\title{
Robust Face Recognition Using Enhanced Local Binary Pattern
}

\author{
Srinivasa Perumal R. ${ }^{1}$, Nadesh R. K. ${ }^{2}$, Senthil Kumar N. C. ${ }^{3}$ \\ School of Information Technology and Engineering, VIT University, India
}

\begin{tabular}{l} 
Article Info \\
\hline Article history: \\
Received Nov 21, 2017 \\
Revised Jan 22, 2018 \\
Accepted Feb 06, 20 \\
\hline Keywords: \\
Face recognition \\
Feature extraction \\
Local binary pattern \\
Local descriptor
\end{tabular}

Article Info

Article history:

Received Nov 21, 2017

Revised Jan 22, 2018

Keywords:

Face recognition

Local binary pattern

\begin{abstract}
Face recognition is an emerging research area in recognition of the people. A novel feature extraction technique was introduced for robust face recognition. Enhanced Local binary pattern (EnLBP) divided the image into sub regions. For each sub region, the salient features are extracted by obtaining the mean value of each sub region. In LBP, each pixel was replaced by applying LBP into each sub region. In this paper, the mean value of sub region was replaced for the sub region. It reduced the dimension of the image and extracts the salient information on each sub region. The extracted features are compared with similarity measures to recognize the person. EnLBP reduces the operation time and computational complexity of the system. The experimental results were carried out in the standard benchmark database LFW-a. The proposed system achieved a higher recognition rate than other local descriptors.
\end{abstract}

Copyright $\odot 2018$ Institute of Advanced Engineering and Science. All rights reserved.

\section{Corresponding Author:}

Srinivasa Perumal R,

School of Information Technology and Engineering,

VIT University, India

Email: r.srinivasaperumal@vit.ac.in

\section{INTRODUCTION}

Face recognition [13] is a challenging task in the unconstrained environment. It is being successfully used in day to day routine activities in major security departments like airport, railway station, central bureau of investigation, etc. Every Human has an ability to remember hundreds of faces that they come across every day which is considered to be a flabbergasting skill of human. But it became increasing onerous for humans to remember all the faces accurately that they had seen years ago. To overcome this issue, automatic face recognition is used for detecting and recognizing the accurate person. When compared with some biometric recognition systems like iris recognition, fingerprint recognition, speech recognition, etc., Face recognition is most acceptable and veracious field of recognition but it suffers from certain issues [14].

The certain factors are illuminations, facial expressions, with different conditions and different poses. The challenging issues are addressed by various approaches such as Principal Component Analysis (PCA) [9], Linear Discriminant Analysis (LDA) [3], Independent Component Analysis (ICA) [2] and Local Binary Pattern (LBP) [6]. The above approaches have achieved better results in constrained environments. In the unconstrained environment, the approaches have achieved a recognition rate, which is less than $50 \%$. To improve the recognition rate, the proposed system is introduced.

The proposed system extracts the local features of the person using an enhanced local binary pattern from the face image. Extracted features are stored in the database. For a query image, the local features are extracted and it matches with the database to recognize the person. LFW-a is the recent challenging face recognition database [6] which consists of 13,233 images with a lot of discrepancies in terms of image quality, illuminations, age as well as the images with variant poses.

The rest of the paper is organized as follows: Section 2 discusses the existing system, Section 3 explains the process of LBP and its functionalities, Section 4 describes the proposed system and Section 5 presents the experimental results of the system and Section 6 concludes the work. 


\section{LITERATURE REVIEW}

Principal Component Analysis (PCA) [9] is used to find vectors that are adequate for the distribution of face images within the entire image space. It also reduces the dimensions with minimal loss in the datasets processed. In PCA, the Eigenvectors and Eigenvalues are computed and Eigenvectors are labeled as feature vectors of the face image. PCA has an advantage of being used in locating and detecting faces, motion detecting and head tracking and also includes the concept of "face spaces" which further helps in recognizing new faces. It also addresses some issues such as scale (head size) and orientation invariance, distance in face space and multiple views. It has drawbacks towards the issues of robustness to its own advantages.

Fisher's [3] proposed linear discriminant analysis (LDA) method, which maximizes the ratio between class scatter matrices and minimizes the ratio within class scatter matrices. LDA reduces the high dimensional vector space into low dimensional vector space. LDA uses labeled set of faces for the training set and unlabeled sets for the testing set and identifies the correct image matching the input. The advantage of the Fisher face method is that, it requires lesser computation time and lower error rates compared to PCA. It achieves good results under lighting issues in the image. This method is insensitive towards large variation in lighting and expression. Independent Component Analysis (ICA) method [2] is a similar form of PCA. PCA employs high order and second order moments of the data and hence depends on Gaussian features even though ICA exploits inherently non-Gaussian features of the data and employs higher order moments. ICA is better than PCA in case of images which are captured at a later time and different facial expressions. ICA reconstructs the image in a better way, even if the image is noisy.

Laplacian faces method [4] is an approach to face analysis, which explicitly considers the manifold structure. This structure preserves the local structure of the unit space using the neighbor graph. Face subspace is obtained by LPP (Locality Preserving Projection) method. The transformation matrix is computed which maps each face image, in the image space to low dimensional subspace called Laplacian Faces. Some nonlinear techniques have been proposed to discover the nonlinear structure of the manifold. The image is projected into PCA subspace having only highest principle components. The nearest neighbor is constructed in an approximation of the local manifold structure. The Laplacian method has discriminating power, although it is unsupervised. It can be applied to any new data point to locate it in a reduced representation space. It can identify a person with different expressions, poses and lighting condition. The drawback is to estimate the intrinsic dimensionality of the nonlinear face manifold. They have not considered the use of unlabeled samples and it is computationally expensive.

Local Binary Pattern [6] introduced for texture description and it is used for face recognition. LBP is the simplest method and convenient technique used for encoding the features for recognition. The LBP is robust in terms of rotation in variant and against monotonic grey level changes. Many researchers extended the local binary pattern to improve the recognition rate. Multi-Block LBP Representation [10], used a set of rectangular features which is distinctive to encode the features of the face image. It encodes the features using LBP that could capture more information about the structure of the image. The encoded features are obtained by calculating traditional LBP approach instead of the actual value, the mean values of the blocks are considered. Each block is divided into $2 \times 3$ regions and is called as sub image and compared with the mean value of the central rectangular block element.

Multi region prominent LBP used to represent the texture of the face by dividing the region into multiple regions to determine the prominent discriminant features [16]. Sabina and Rana introduced soft local binary pattern (SLBP) for face recognition if the images in noisy condition with the help of edge detection techniques [17]. Ma et al., proposed random projection based feature extraction for face recognition [5]. It is a simple and effective method, but less robustness in occlusion and disguise. Modular Two Dimensional PCA addressed the limitations in an uncontrolled environment [18]. It divides the image into four regions and applied the histogram equalization to all the regions for reducing the illumination of the region to satisfy the lightening conditions. Dimensionality reduced local directional pattern (DR-LDP) introduced to reduce the dimension of the feature space. It divides the image into several regions and it extracts the feature of the each region to form a single feature for a region. DR-LDP and MB-LBP recognition rate motivates us to introduce a novel descriptor with reduced dimension. Recently extended and median based LBP was introduced to describe the texture of the face to recognize the person [7], [8].

\section{LOCAL BINARY PATTERN}

Local binary Pattern (LBP) [6] is a descriptor for texture description. LBP divides the image into size of 3 X 3 regions. LBP extracts the important feature from each region. In each region, the center pixel value is compared with its 8 neighbors, if the center pixel value is less than the neighbor value, then the neighbor position is set as 0 otherwise it is set as 1 . Ahonen et al. used LBP operator to describe the face 
description [1]. The face image is divided into 3 X 3 overlapped regions. For each pixel value, a new eight bit code is assigned based on the center pixel and its neighbor in the $3 \times 3$ overlapped regions. The resultant matrix is the LBP representation of the face image. The LBP encoded image is divided into 8 X 8 regions and apply the histogram to extract the features of each region. Histogram of the region contains discriminate information of the face.

Each region of the histogram features are concatenated into a single histogram feature. The dissimilarity measure is used to perform the recognition task. LBP is robust against pose, facial expression, and lighting. Also, it is computationally efficient and simple. The LBP is defined in equation 1. Figure 1 shows the computational steps of local binary pattern.

$$
\operatorname{LBP}_{P, R}\left(x_{c}, y_{c}\right)=\sum_{p=0}^{p-1} S\left(g_{p}-g_{c}\right) 2^{p}
$$

where,

$$
s(x)= \begin{cases}1, & x \geq 0 \\ 0, & x<0\end{cases}
$$

where $\mathrm{P}$ represents the number of sampling points on a circle of radius $\mathrm{R}$.

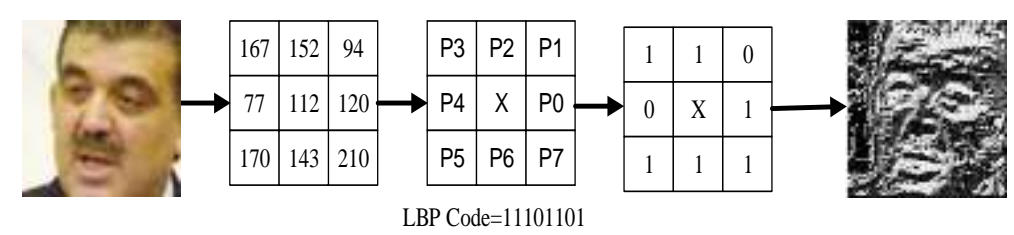

Figure 1. Computational steps of local binary pattern

\section{ENHANCED LOCAL BINARY PATTERN}

Enhanced Local Binary Pattern (EnLBP) divides the image into 3 X 3 regions. In each $3 \times 3$ regions, the neighboring pixels are added with center pixel and the mean value is computed to extract local information of the regions. The mean value of the matrix is computed and stored as a vector for a region. The center pixel is compared with its 8 neighboring values in the resultant image to reduce the dimension of the image. If the center pixel value is less than its neighboring value, the corresponding neighboring positions are set as 1 else 0 . The 8 bit binary values are taken in a sequence and it converts into decimal value. The decimal value is substituted to the center position of the region. Algorithm 1 and Figure 2 demonstrates the procedure of Enhanced Local binary pattern.

\section{Algorithm: EnhancedLBP \\ Input: Original image \\ Output: EnLBP encoded image}

1. For the given input image, obtain EnLBP encoded image based on equation 1.

2. Divide the encoded image into $3 \times 3$ non-overlapping regions.

i) For each region, the binary code is computed by the neighborhood of the center pixel.

ii) Apply arithmetic operation to sum the neighbors of the center pixel to form a single eight bit binary code for the $3 \times 3$ region.

iii) Convert the resultant binary code $\mathrm{p}$ into an equivalent decimal value.

3. Repeat the steps 2 to reduce the dimension of the image.

EnLBP extracts the local information of the image to improve the performance. When an image of higher dimensions is fed as an input for matching, it becomes complex to deal with the large image as it consumes more time for computation. To overcome this issue, EnLBP is introduced for dimensionality 
reduction without losing any salient features. EnLBP is mainly used to reduce the dimension and the operation time of the system.

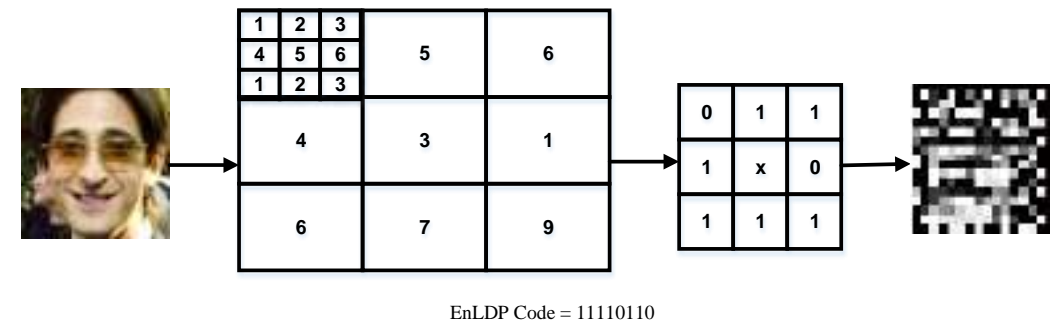

Figure 2. Procedure of enhanced local binary pattern

The proposed EnLBP method uses the salient features of the face for recognition. The images are trained by EnLBP and it is stored in the database. The query image is chosen from the testing set and it extracts the features using EnLBP. Finally the feature of query image is compared with features stored in the database. To determine the similarity of the query image and the stored image, the cosine distance measures are used to recognize the face. The query image is recognized with a stored image in the database by following equation (2).

$$
\text { Similarity }=\operatorname{Cosine}(\theta)=\frac{A \cdot B}{\|A\| \cdot\|B\|}
$$

where, $A_{i}$ is the query image matrix from the testing set and $B_{i}$ is the reduced training set image matrix. Each $A_{i}$ image matrix is compared with the entire image in $B$ matrix. For example, while computing the distance formula for an input image, we need to consider all the training set images, say (70). The query image is compared with all 70 images and the distance of the image which is near to 1 is considered as found image.

\section{RESULTS AND ANALYSIS}

The benchmark dataset used in the method is LFW-a, which contains a stock of labelled images that could be used for image processing in an unconstrained image [11], [12]. LFW-a is also similar to LFW datasets except that the images in LFW-a are aligned using a commercial alignment software.

The alignment is done for the sake of improving the performance of face recognition algorithm. The experiments are accomplished using Intel i5 processor at $2.60 \mathrm{GHz}$. LFW-a database is used, because it is the most challenging dataset in an unconstrained environment. It consists of images, variations in pose, lighting, quality, etc. In LFW-a, there are about 1056 images of 433 individuals. The system randomly considers the images from the set of individuals which are distinct from one another in terms of pose, expression, background color, lighting, frontier view, etc. From the 433 individual images, those individuals containing more than 10 images are considered as a training set by the system. For each individual, 5 images are chosen randomly and it considered as train set and remaining images are considered as test set.

EnLBP method has been deployed over LFW-a, 70 images are chosen randomly from 14 individuals, which consists of more than 10 images of each person in the database, and it is used for matching. The system performance rate is $61.428 \%$, which is significantly higher in comparison to $56.75 \%$ in the existing system [15].The existing system used local binary pattern technique in LFW database. In the system, extension to the techniques and improvisation to the efficiency of the face recognition process have been performed. The average recognition rate, accuracy rate, and error rate of Enhanced Local Binary pattern comparison with other methods is shown in the following Figure 3. 


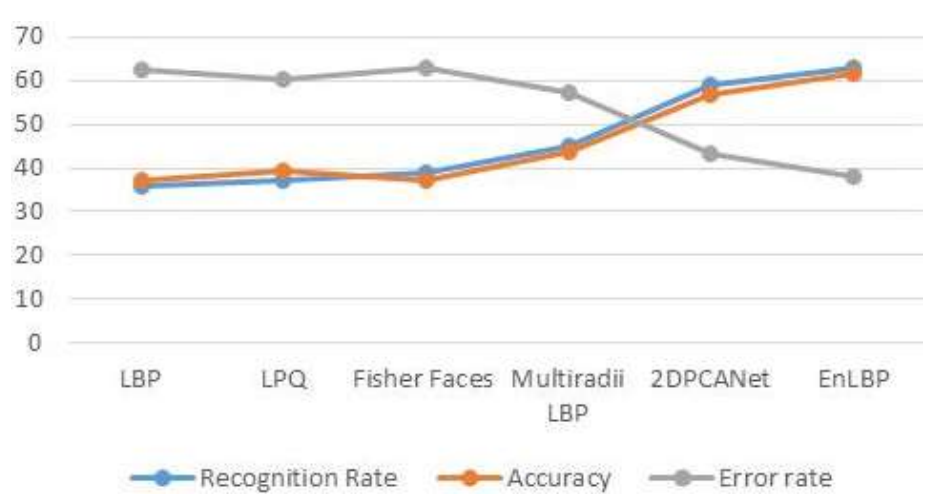

Figure 3. Comparision results of EnLBP with other existing methods

The ORL database contains 40 different persons image. Each subject 10 images with different variation (varying the lighting, facial expressions and facial details). Each subject five images are randomly selected for training and rest 5 images for testing. In ORL database 200 images are used as gallery images and remaining 200 images are used as probe set. The recognition rate of the proposed system is compared with existing holistic approaches and is shown in Table 1.

Table 1: Comparison Results of EnLBP with Other Existing Methods

\begin{tabular}{cc}
\hline Method & Recognition Rate \\
\hline LBP & 97.15 \\
LPQ & 97.87 \\
Fisher Faces & 96.45 \\
Multiradii LBP & 98.67 \\
2DPCANet & 99.45 \\
EnLBP & 99.07 \\
\hline
\end{tabular}

The result shows that the 2DPCANet attained a better recognition rate than the proposed method. The computational time of the proposed method is less than the 2DPCANet. 2DPCAnet required more space for computation and the recognition rate is proportionally equal.

\section{CONCLUSION}

The descriptor divides the image into $3 \times 3$ sub regions for extracting the local information of the image. The proposed method is an efficient method in partial image and occluded image. The results show that the system is a robust on comparison to the previous techniques for face recognition. The experimental results on LFW-a database are less than $50 \%$ attained by the existing systems. The method achieved a higher recognition rate of $61.43 \%$ compared with other existing systems. However, this method has been performed only over LFW-a database, it could be further applied on various other databases such as CMU, AR etc. in further works.

\section{REFERENCES}

[1] Ahonen, T., Hadid, A., \& Pietikainen, M. Face description with local binary patterns: Application to face recognition. IEEE Transactions on Pattern Analysis and Machine Intelligence. 2006; 28(12): 2037-2041.

[2] Bartlett, M. S., Movellan, J. R., \& Sejnowski, T. J. Face recognition by independent component analysis. IEEE Transactions on Neural Networks. 2002; 13(6): 1450-1464.

[3] Belhumeur, P. N., Hespanha, J. P., \& Kriegman, D. J. Eigenfaces vs. fisherfaces: Recognition using class specific linear projection. IEEE Transactions on Pattern Analysis and Machine Intelligence. 1997; 19(7): 711-720.

[4] He, X., Yan, S., Hu, Y., Niyogi, P., \& Zhang, H. J. Face recognition using Laplacianfaces. IEEE transactions on Pattern Analysis and Machine Intelligence. 2005; 27(3): 328-340.

[5] Ma, C., Jung, J. Y., Kim, S. W., \& Ko, S. J. Random projection-based partial feature extraction for robust face recognition. Neurocomputing. 2015; 149: 1232-1244.

[6] Ojala, T., Pietikäinen, M., \& Harwood, D. A comparative study of texture measures with classification based on featured distributions. Pattern Recognition. 1996; 29(1): 51-59. 
[7] Liu, $\mathrm{Li}$, et al. Median robust extended local binary pattern for texture classification. IEEE Transactions on Image Processing. 2016; 25(3): 1368-1381.

[8] Liu, Li, et al. Extended local binary patterns for face recognition. Information Sciences. 2016; 358: 56-72.

[9] Turk, M. A., \& Pentland, A. P. Face recognition using eigenfaces. IEEE Computer Society Conference on Computer Vision and Pattern Recognition, 1991; 586-591.

[10] Zhang, L., Chu, R., Xiang, S., Liao, S., \& Li, S. Z. Face detection based on multi-block lbp representation. International Conference on Biometrics. 2007; 11-18.

[11] Huang, G. B., Mattar, M., Berg, T., \& Learned-Miller, E. Labeled faces in the wild: A Database for Studying Face Recognition in Unconstrained Environments. In Workshop on faces in'Real-Life'Images: detection, alignment, and recognition. 2008.

[12] Learned-Miller, E., Huang, G. B., RoyChowdhury, A., Li, H., \& Hua, G. (2016). Labeled Faces in the Wild: A Survey. In Advances in Face Detection and Facial Image Analysis. 2016; 189-248.

[13] Gross, R., Baker, S., Matthews, I., \& Kanade, T. Handbook of Face Recognition, 2005: 193-216.

[14] Zhao, W., \& Chellappa, R. Face Processing: Advanced modeling and methods. Academic Press. 2011.

[15] Yu, D. \& Wu, XJ. 2DPCANet: a deep learning network for face recognition, Multimedia Tools Applications, 2017. https://doi.org/10.1007/s11042-017-4923-3.

[16] Konda SR, Kumar V, Krishna V. Face Recognition using Multi Region Prominent LBP Representation. International Journal of Electrical and Computer Engineering (IJECE). 1 Dec 2016; 6(6): 2781.

[17] Yasmin S, Rana MM. Performance Study of Soft Local Binary Pattern over Local Binary Pattern under Noisy Images. International Journal of Electrical and Computer Engineering (IJECE).1 Jun 2016; 6(3): 1161.

[18] Venkatramaphanikumar S, Kishore KK. Face Recognition with Modular Two Dimensional PCA under Uncontrolled Illumination Variations. International Journal of Electrical and Computer Engineering (IJECE). 1 Aug $2016 ; 6(4): 1610$.

[19] Srinivasa Perumal R, Chandra Mouli PVSSR. Dimensionality reduced local directional pattern (DR-LDP) for face recognition. Expert Systems with Applications. 2016 Nov 30;63:66-73. 